\title{
The seizure semiology consistent with frontal lobe symptomatogenic zone in children
}

\author{
Ülkühan Öztoprak¹, Dilek Yalnızoğlu², Kader Karlı Oğuz³, Eser Lay Ergun ${ }^{4}$, Figen \\ Söylemezoğlư ${ }^{5}$, Burçak Bilginer ${ }^{6}$, Nejat Akalann${ }^{6}$, Meral Topçu² $^{2}$ Güzide Turanl1 ${ }^{7}$ \\ ${ }^{1}$ Department of Pediatric Neurology, Dr. Sami Ulus Maternity and Childrens' Health and Diseases Training and Research \\ Hospital, Ankara; ${ }^{2}$ Division of Pediatric Neurology Deparment of Pediatrics, Hacettepe University Ihsan Dogramacr \\ Children's Hospital, ${ }^{3}$ Deparment of Radiology, ${ }^{4}$ Deparment of Nuclear Medicine, ${ }^{5}$ Deparment of Pathology, ${ }^{6}$ Deparment of \\ Neurosurgery, Hacettepe University Faculty of Medicine, Ankara; ${ }^{7}$ Division of Pediatric Neurology, Deparment of Pediatrics, \\ Istanbul Medipol University, Istanbul, Turkey. \\ E-mail: ulkuhantoprak@yahoo.com \\ Received: 2nd September 2016, Revised: 15th November 2016, Accepted: 15th February 2017
}

SUMMARY: Öztoprak Ü, Yalnızoğlu D, Karlı-Oğuz K, Lay-Ergun E, Söylemezoğlu F, Bilginer B, Akalan N, Topçu M, Turanlı G. The seizure semiology consistent with frontal lobe symptomatogenic zone in children. Turk J Pediatr 2016; 58: 583-591.

The aim of this study is to analyze the seizure semiology consistent with frontal lobe symptomatogenic zone in childhood.

We analyzed 549 videotaped seizures from 79 patients (mean age $9.9 \pm 3.8$ years). Magnetic resonance imaging was normal in 30 patients. The seizures in the time interval of $10 \mathrm{p} . \mathrm{m}$. to $6 \mathrm{a} . \mathrm{m}$. were considered as nocturnal.

The mean number of seizures per patient was $6.8 \pm 7.3$. The mean seizure duration was $25.7 \pm 26.9 \mathrm{sec}$; postictal confusion was $27 \pm 16.1 \mathrm{sec}(7-92$ seconds). The seizures were observed in sleep with a rate of $56.8 \% ; 43.1 \%$ of them were during wakefulness. Overall $50.4 \%$ of the seizures occured during night-time sleep. Tonic seizure $(\mathbf{7 7 . 2 \% )}$ was the most frequent simple motor seizure. Versive seizures were the second most frequent type of simple motor seizure $(26.7 \%)$. Clonic seizures were $17.7 \%$, complex motor seizures were $20.5 \%$, and dialeptic seizures were $3 \%$ of all the seizures. Epileptic spasm, myoclonic seizures, aphasia, and akinetic semiologies were not observed. Vocalization was observed in $16 \%$ of the seizures.

Frontal lobe seizures in childhood have a short duration, occur frequently, especially during night time sleep, and have a brief postictal period. Tonic semiology, versive semiology are the most frequent seizure semiologies; hypermotor and secondary generalized tonic clonic seizures and vocalizations are observed less in children compared to adults.

Key words: frontal lobes, seizure semiology, childhood.

In childhood, extratemporal lobe epilepsy is more common than temporal lobe epilepsy compared to adults; with majority of seizures generating from frontal lobes. Patients who had seizures originating from frontal lobes constitute $14-20 \%$ of patients who underwent epilepsy surgery for the management of intractable seizures ${ }^{1-3}$.

In literature semiological features of frontal lobe seizures are documented in patients, mostly adults, who underwent invasive recordings and cortical stimulation, and achieved seizure freedom following frontal resections. Distinctive features of frontal lobe seizure include a short duration, a high daily frequency, bizarre complex motor automatisms, vocalization, rapid and frequent secondary generalization, prominent and early motor manifestation ${ }^{4-6}$. Although, most of the drug-resistant partial epilepsies are generated from frontal lobes in childhood, information on seizure semiology in frontal lobe seizures in children are sparce. The semiological seizure classification (SSC) describes the somatotopic distribution of 
the ictal semiology as well as the seizure evolution defined by witnesses, patients or as observed on video-monitoring. EEG findings or neuroimaging findings are not taken in consideration in this classification.

We reviewed seizures consistent with frontal lobe symptomatogenic zone according to SSC in children and analyzed the distribution of subtypes and diurnal variation.

\section{Material and Methods}

\section{Patients}

We reviewed 332 patients admitted to longterm video-EEG monitoring unit at Hacettepe University Children's Hospital between January 2007 and December 2009. Ninety-seven patients out of 332 (29.2\%) were diagnosed as frontal lobe seizures; 79 patients with adequate data were analyzed. Eighteen patients were excluded as:

The patient was either off camera or was covered so that the semiological symptoms could not be fully visualized. We excluded these cases even if they had recorded seizures on video-EEG were not able to document all seizure types in these patients.

The patients had merely electrographic seizures originating from frontal area.

We studied patients with seizure semiology consistent with frontal lobe symptomatogenic zone and scalp EEG findings supportive of ictal onset from frontal lobe.

\section{Long-term video-EEG}

Long-term video-EEG recordings were performed with scalp electrodes placed according to the 10 - 20 system. A companion stayed with the patient in order to report the behavior changes and seizures via an alarm button. The patients were examined by trained nurses and/or doctors during and after the seizures to assess the level of consciousness and presence of motor deficits. The recorded videos of the seizures were analyzed by the authors (UÖ, GT) who were not blind to the patients.

The seizures were classified according to the 'Semiological Seizure Classification'7. All semiological subtypes were analyzed starting from the initial clinical and EEG change until the end of the seizures. The evolution of the seizures was limited by three semiological subtypes. The occurence of the seizures were distinguished as during sleep and awake. The seizures in the time interval of 10 p.m. to 6 a.m. were considered as nocturnal. The videorecordings that reveal 5 minutes following the cessation of seizures were evaluated in order to assess postictal clinical symptoms.

We defined vocalizations as making sounds other than comprehensible speech, apnea, or sounds due to muscle contractions during clonic or tonic clonic seizures. The clinical and neuroimaging data were obtained from epilepsy follow-up sheets at the video-EEG monitoring unit and from medical charts of the patients. The age at the time of initial seizures, onset of recurrent seizures, history of febrile seizures, family history of febrile/afebrile seizures, results of physical and neurological examination and MRI findings were reviewed. Approval for this study was granted by the Hacettepe University Medical Faculty Ethical Committee (number: 431.10-424).

\section{Statistical Analysis}

SPSS 14.01 (Serial: 9869264) program was utilized for statistical analysis. Chi-square test and descriptive statics were used; $p<0.05$ value was considered statistically significant. The compliance rate between the two variables were obtained by evaluating the Kappa compliance rate and total accuracy rate.

\section{Results}

\section{Clinical data}

We analyzed 79 patients between 1-17 years of age (mean $9.9 \pm 3.8$ years); $43(54.4 \%)$ were boys, and $36(45.6 \%)$ girls. Sixty-three $(79.7 \%)$ patients were 7 years and older and 16 patients $(20.3 \%)$ were younger than 7 years.

The age at time of the initial seizure was between 1 month and 15 years (mean 38.07 \pm 43.7 months). In $41.8 \%$ of the patients the first seizure occured during in the first years of life. In $35.4 \%$ of the patients recurring seizures started in the first year of life. Fifteen (18.9\%) of the patients had history of febrile seizures, $20 \%$ had family history of epilepsy or febrile seizures, $27.8 \%(22 / 79)$ had hemiparesis. $83.5 \%(66 / 79)$ of the patients were taken on polytherapy; during the admission antiepileptic drugs were decreased in $10(12.6 \%)$ patients, discontinued in $13(16.4 \%)$ patients, and remained the same in $56(70.8 \%)$ patients. 
Magnetic resonance imaging (MRI) was normal in 30 patients, showed a focal abnormality in 49 patients; MRI showed unilateral lesions in 38 patients, bilateral abnormalities in 11 patients either involving merely frontal lobe(s) or extending beyond frontal lobes. MRI abnormalities consisted of $22.7 \%$ cortical dysplasia, $19 \%$ lesions due to sequela of stroke or intracranial bleeding, $8.8 \%$ primary brain tumors and $6.3 \%$ phacomatoses (Table I).

Seven $(8.9 \%)$ patients had epilepsy surgery prior to admission and were re-evaluated for ongoing seizures; 3 had resective surgey (2 frontal resections, 1 anterior temporal lobectomy), 2 had functional hemispherotomy, 1 had 2/3 anterior corpus callosotomy, 1 had implantation of vagal nerve stimulation.
Seventeen (\% 21.5) patients were admitted for phase 1 presurgical evaluation and 10 patients subsequently underwent resective surgery ( 6 on the right, 4 on the left frontal lobe), 4 patients had implantation of vagal nerve stimulation, functional hemispherotomy was performed on 3 patients. The follow-up duration after epilepsy surgery was $19.3 \pm 12.8$ months (2-40 months). In $60 \%$ of the patients $(6 / 10)$ who had resective surgery the etiology was focal cortical dysplasia, $47.1 \%(8 / 17)$ of the patients were seizure free (Engel Class 1), $11.8 \%$ Class II, $23.5 \%$ Class III, $17.6 \%$ had Class IV outcome.

\section{Seizures Characteristics}

We analyzed 549 seizures of 79 patients. The mean number of seizures per patient was

Table I. The MRI Findings of Children with Frontal Lobe Seizures.

\begin{tabular}{lll}
\hline Brain MRI & $\mathrm{n}$ & $(\%)$ \\
\hline Normal & 30 & 38.0 \\
Malformations of cortical development & 18 & 22.7 \\
& (a) Right frontal lobe dysplasia : 8 \\
& premotor cortex (1), primary motor cortex (1) \\
& (b) Left frontal lobe dysplasia : 7 \\
& orbitofrontal cortex (1), primary motor cortex (1) \\
& (c) Bilateral frontal lobe dysplasia: 3
\end{tabular}

Stroke, ischemic/hemorrhagic lesions

Primary brain tumor

(a) Right frontal lobe tumor: 3

anterior cingulate cortex (1)

(b) Left frontal lobe tumor : 4

left motor cortex (1)

left sensorimotor cortex (1; DNET)

Phakomatoses

5

Tuberosclerosis (4)

Sturge-Weber syndrome(1)

Asymmetric hippocampal head with malrotation 1

on the right

\begin{tabular}{lll} 
Periventricular leukomalacia & 1 & 1.3 \\
Diffuse cortical atrophy & 1 & 1.3 \\
Post traumatic changes & 1 & 1.3 \\
\hline Total & 79 & 100
\end{tabular}

DNET: dysembryoplastic neuroepithelial tumor; MRI: magnetic resonance imaging 
$6.8 \pm 7.3$ (1-46 seizures). The mean seizure duration was $25.7 \pm 26.9 \mathrm{sec}(2-244 \mathrm{sec})$. With respect to seizure occurrence, 312 of 549 seizures $(56.8 \%)$ were observed in sleep, 237 of them $(43.1 \%)$ were during wakefulness, 328 of them $(59.7 \%)$ were at night, 221 of them $(40.3 \%)$ were during day time. When the occurrences of the seizures were analyzed with respect to wakefulness, sleep and circadian patterns (diurnal/nocturnal), 50.4\% (277/549) of the seizures were seen in night-time sleep $(\mathrm{p}<0.05)$.

In $57.1 \%(314 / 549)$ of the seizures, only one semiological sub-type was observed whereas two or three semiological sub-types were observed in $42.9 \%$ of the seizures.

Tonic seizure was the most frequent simple motor seizure $(77.2 \%)$. Fifty percent of the tonic seizures were focal tonic, $43.3 \%$ were asymmetric tonic and $6.6 \%$ were generalized tonic (Table II).

Versive seizures were the second most frequent type of simple motor seizure $(26.7 \%) ; 55.1 \%$ of versive seizures were observed as the initial clinical behavior of the seizures, $31.2 \%$ ocurred during the seizure evolution, $13.6 \%$ of them ocurred before secondary generalized tonic clonic seizures. Clonic seizures were $17.7 \%$ of all the seizures; $81.4 \%$ showed focal clonic semiology and $17.5 \%$ of them were generalized clonic. Secondary generalized tonic-clonic seizures (SGTC) constituted 6.3\% of the seizures.

Complex motor seizures were seen in $20.5 \%$ of all seizures. Hypermotor semiology was observed in $95 \%$, and $5 \%$ were gelastic seizures. Majority (75\%) of hypermotor seizures were seen as the first symptom of seizures. Automotor seizures with distal automatisms were not observed. Dialeptic seizures were 3\% of all the seizures. All dialeptic subtypes were seen as the first symptom of seizures. Aura was the least common subtype present in $1 \%$ of the seizures, and seen only in one patient. Aura was classified as psychic aura in all 6 seizures with fear. Atonic semiology, one of the special seizures, was also rare seen in only $1 \%$ of the seizures. Epileptic spasm, myoclonic seizures, aphasia, akinetic semiologies were not observed. Vocalization was observed in $16 \%$ of all the seizures. Semiologic findings across different ages were summarized in Table III.

During the postictal period, assessment of level of consciousness was performed in $71.7 \%$ of all the seizures, motor strength in $74.4 \%$, and speech in $70.1 \%$. Postictal confusion was present in $29.9 \%$, Todd paralysis in $10.7 \%$, and the postictal aphasia in $2 \%$. In $4.8 \%$ of the seizures emotional changes such as crying, and agression were noted following the seizure. The duration of postictal confusion ranged between 7-92 seconds (mean $27 \pm 16.1$ seconds).

Table II. Distribution of Semiologic Findings* in Children with Frontal Lobe Seizures.

\begin{tabular}{lcc}
\hline \multicolumn{1}{c}{ Semiologic findings } & Patients, $(\mathrm{n}:$ 79), $[\mathrm{n}(\%)]$ & Seizures $(\mathrm{n}: 549),[\mathrm{n}(\%)]$ \\
\hline Aura & $1(1.3)$ & $6(1)$ \\
Dialeptic seizure & $6(7.6)$ & $16(3)$ \\
Simple motor seizure & & \\
Tonic & $62(78.5)$ & $424(77.2)$ \\
Versive & $39(49.4)$ & $147(26.8)$ \\
Clonic & $31(39.2)$ & $97(17.7)$ \\
Tonic-clonic & $16(20.3)$ & $35(6.3)$ \\
Complex motor seizures & $28(35.4)$ & $108(19.6)$ \\
Hypermotor & $1(1.3)$ & $5(1)$ \\
Gelastic & $4(5.1)$ & $6(1)$ \\
Special seizures & & \\
Atonic & &
\end{tabular}

${ }^{*} \geq 2$ semiologic subtypes were seen in $\% 42.9$ of seizures and in $\% 44.3$ of patients 
Secondary generalized tonic clonic seizures had longer postictal confusion.

\section{Discussion}

Clinical features of frontal lobe seizures were studied in patients, mostly in adult population, who underwent video-EEG monitoring, invasive electrophysiologic studies, cortical stimulation, and who achieved freedom following frontal resections. None of our patients underwent such evaluation, therefore we are not able to determine whether the seizures originated from frontal lobes. The limitation of our study is that our patients exhibit the semiological features consistent with frontal lobe symptomatogenic zone, which is supportive of involvement of frontal lobe during the seizure, however it is not clear whether the seizures originated from frontal lobes or the symptomatology was due to propagation from other areas.

In adults, frontal lobe seizures last less than one minute and daily seizure frequency is very high ${ }^{8,9}$. The average time of seizures was found to be 29 seconds in a study which investigated frontal lobe semiology in children under 7 years $^{10}$. In our study, the number of seizures per patient was on average 6.8 ; and the duration was $25.7 \mathrm{sec}$ in accordance with previous reports 11,12 .

Frontal lobe seizures are generally related to sleep ${ }^{13,14}$. Loddenkemper et al. ${ }^{15}$ studied children with lesional focal epilepsy, and showed that frontal and parietal lobe seizures occurred mostly during sleep independent of day or night. Gurkas et al. ${ }^{16}$ described 170 patients (909 seizures) and found that frontal lobe seizures occurred more often at night and in sleep. In our study, the occurence of seizures were grouped with respect to sleep and awake states, as well as day and night-time sleep. Overall $50.4 \%$ of the seizures occurred during night-time sleep. We concluded that sleep, especially night-time sleep, may provoke frontal lobe seizures in childhood more than any state.

Epilepsy in childhood differs widely from adults ranging from the etiology, clinical presentation, developmental problems, cognitive functions, treatment and prognosis. Developmentally the most significant interval that makes a striking difference between adult and childhood epilepsy is the newborn period and infancy ${ }^{17}$. The semiological features of temporal lobe seizures have been studied widely, semiological features suggestive of temporal lobe start to emerge as early as three years old, however more specific semiological features such as aura, automatisms, dystonic posturing and secondary generalization are seen over 6 years, bilateral symmetric tonic semiology decreases with advanced age ${ }^{18}$. In our series aura was seen in only one patient who was older than 7 years of age. The majority of patients with dialeptic seizures were 7 years and older. Motor seizures, especially hypermotor and versive seizures were again mostly seen in patients 7 years and older. Notably, a single type of seizure predominated the semiology in patients 7 years and older.

Table III . Distribution of Seizure Semiology with Respect to Age in Children with Frontal Lobe Seizures.

\begin{tabular}{lcc} 
Semiology (n) & $<7$ years, n $(\%)$ & $\geq 7$ years, $\mathrm{n}(\%)$ \\
\hline Aura (1) & 0 & $1(100)$ \\
Dialeptic (6) & $2(34)$ & $4(66)$ \\
Tonic (62) & $15(24)$ & $47(76)$ \\
Clonic (31) & $10(32)$ & $21(68)$ \\
Versive (39) & $5(13)$ & $34(87)$ \\
Tonic-clonic (16) & $3(19)$ & $13(81)$ \\
Hypermotor (28) & $2(7)$ & $26(93)$ \\
Gelastic (1) & 0 & $1(100)$ \\
Atonic (4) & $1(25)$ & $3(75)$ \\
Mixed types of seizures (35) & $12(35)$ & $23(75)$ \\
\hline
\end{tabular}


In our study, mixed type seizures, that is presence of more than one type of seizure, were seen in children younger than 7 years of age. Bilateral asymmetric tonic semiology was also seen mostly in this age group. On the other hand focal tonic seizures, aura and dialeptic semiology were seen in children 7 years and older. The occurrence of different seizure semiologies in the same patient may be related to frontal lobe immaturity rather than presence of independent foci.

Fogarasi et al. ${ }^{1}$ compared the frontal and posterior cerebral lob seizure semiologies in 35 children, it was concluded that tonic semiology was more common in frontal lobe epilepsies. Tonic seizures occur in $8-38 \%$ of adult patients with frontal lobe epilepsy8,19,20. Bonini et al. ${ }^{21}$ studied 54 patients diagnosed frontal lobe epilepsy (mean age at recording was $24.9 \pm 9.5$ ) and were examined by stereoelectroencephalography (SEEG) during presurgical evaluation and found contralateral tonic posture were caused by precentral and premotor regions. In our study, the most frequent simple motor seizure was tonic seizure $(77.2 \%)$ and of $50 \%$ were focal tonic. We conclude that tonic semiology may be a signiture finding for frontal lobe seizures in childhood.

Versive seizures have been considered as semiological symptoms specific to frontal lobe epilepsies in adults, and were thought to be related to occipital lobe seizures in children ${ }^{1,22}$. However, semiological studies in children have shown that versive seizures are seen in frontal lobe seizures ${ }^{12,} 23,24$. In our study, versive semiology was the second most common seizure type in accordance with this information. We observed versive seizures as the single semiology, or as a subtype during the evolution of the seizure. Half of the versive subtype occured as the initial semiology, one thirds occured as the seizure evolved. Of note, $13.6 \%$ of this subtype occured before secondary generalization, which has a high lateralization value and thought to be almost spesific for frontal lobe seizures in adults.

In adults with frontal lobe epilepsy who became seizure free after the surgery, $14-35 \%$ of the patients experienced focal clonic seizures, lateralizing to the contralateral hemisphere in $85-90 \%$ of the seizures ${ }^{25-27}$. In children, $14-$
$30 \%$ of seizures showed focal clonic semiology, lateralizing to the contralateral hemisphere in $90-100 \%$ of the seizures ${ }^{1,12,18}$. In our study, clonic semiology was observed in $17.7 \%$ of the seizures and mostly consisted of focal clonic seizures (81.4\%).

Secondary generalized tonic clonic seizures generally accepted as a typical feature of adult frontal lobe epilepsy, and is reported in 61$91 \%$ of patients $4,20,22$. This ratio varies around $5-30 \%$ in children with frontal lobe epilepsy due to maturation $6,12,24,28$. We similarly observed secondarily generalized tonic-clonic seizures in $20 \%$ of our patients and $6.3 \%$ of the seizures.

Hypermotor seizures are abundantly seen in adult patients with frontal lobe seizures and seizures originating from temporal pole ${ }^{29}$. However, information regarding hypermotor seizures in children with frontal lobe seizures is sparce in the literature. Hypermotor seizures are not reported in children with frontal lobe seizures who are under 7 years attributed to the immaturity of the frontal lobe in this age group ${ }^{10}$, however this semiology is observed in $14 \%$ of frontal lobe seizures in another study involving children of all ages ${ }^{12}$. In this study the age distribution is unclear, and whether patients under 7 years had hypermotor seizures is not addressed. In our study on $35.4 \%$ of patients, $19.6 \%$ of seizures showed hypermotor semiology. Hypermotor semiology was predominantly seen in children 7 years and older, however we also observed this semiology in 2 patients under 7 years.

We have not observed automotor seizures in our patients. In childhood the connection of the frontal lobe with temporomedial structures are less active than the connections with other lobes ${ }^{30,31}$. This might be the reason why we have not observed seizures with propogation to the temporal lobe and showing typical temporal lobe semiology such as automotor seizures. Manford et al. ${ }^{19}$ studied temporal and frontal lobe seizures in adults and found that automotor semiology indicated temporal lobe with statistical significance. We have not observed epileptic spasms, either, this was attributed to age factor. We had only one patient under 2 years of age.

Documentation of auras is difficult in childhood as children may not be able to describe or articulate their symptoms. A video-EEG 
monitoring study in childhood on semiological seizure classification showed that aura was detected in $15.8 \%$ of all seizures and $8.5 \%$ of those were classified as psychic aura ${ }^{23}$. The age of patients with aura was not specified. In another study in children with frontal lobe epilepsy, aura was defined as sudden changes on facial expression or behavior before the seizure or change in the emotional state. Based on this definition of aura, $57 \%$ of the patients were diagnosed with aura ${ }^{32}$. In our study, only the symptoms that were expressed by the patients themselves were accepted as aura. One of our patients had 6 seizures consisting of psychic aura described as a feeling of fear. In our study, the facial expression of fear was accompanied with hypermotor seizures. Interactions of the network connections responsible for emotions, between orbitofrontal lobe and amygdala may be responsible for this clinical semiology 33,34 .

In adult studies on frontal lobe epilepsies ictal vocalization varies from 30 to $70 \%$ of the seizures ${ }^{11,20,35}$. In a study with children younger than 12 years old with lesional frontal lobe epilepsy, $16 \%$ of the seizures showed vocalization $^{12}$. In our study vocalization in the form of shouting and screaming was seen in $16 \%$ of the seizures. Vocalization is another semiological feature seen in frontal lobe seizures represented predominantly in adults, similar to auras and secondarily generalized seizures.

Semiological seizure studies tend to state postictal findings less than individual seizure characteristics. Evaluation of state of consciousness, ability to talk and presence of motor deficits in postictal period, is important and may be critically informative in children as in adults, however it is also much harder. It is often difficult to achieve communication with children during postictal period. Postictal motor deficits is a diagnosis that has a robust lateralizing value; it indicates seizure focus in the contralateral side with $100 \%$ rate $^{27}$, 36,37 . In a study of postictal motor deficit in adults (44 patients with a mean age of 31 years) with focal epilepsies $13.4 \%$ of the seizures were identified as Todd's paresis ${ }^{36}$. In a study conducted in children, postictal Todd's paresis was diagnosed in $14 \%$ of the patients, in another study conducted on 35 pediatric patients only 1 was diagnosed ${ }^{38}$. In our study $24.1 \%$ of the patients, and $11.7 \%$ of the seizures showed Todd's paresis postictally. Postictal Todd's paresis develops following focal motor seizures ${ }^{39}$. In our study, the presence of high percentage of postictal motor deficits may be associated with the high rate of focal motor seizures in our patients.

Frontal lobe seizures in adults are followed by short duration of postictal confusion on average less than 1 minute 9,23 . In our study due to the age factor, it was hard to access the level of consciousness of the patients, however we were able to evaluate 394 seizures regarding postictal level of consciousness. Postictal confusion was present in $30 \%$ of the seizures and lasted 7-92 seconds (mean 27 seconds) starting from the sessation of the seizure. As expected, seizures with longer postictal confusion were secondary generalized tonic clonic seizures. Our results emphasize the importance of examination of patients following seizures, especially with trained nurses.

Malformations of cortical development were the most common cause in our symptomatic cases, followed by brain injury due to perinatal insult. In current literature malformations of cortical development is the most common substrate in children with intractable epilepsy followed by tumors as the second most common etiology. The etiological profile in our patients may have implications regarding preventable factors underlying intractable epilepsy.

We have a selection bias since we selected patients who had seizures consistent with frontal lobe symptomatogenic zone. The evaluation of such a large series enabled us to observe the distribution of different subtypes in this patient population. We were able to use Semiological Seizure Classification in children with frontal lobe seizures effectively. We found this classification practically applicable in children. Frontal lobe seizures in childhood have a short duration, occur frequently, especially during night time sleep, and have brief postictal period. Tonic semiology, especially focal tonic subtype and versive semiology are the most frequent seizure semiologies; hypermotor and secondary generalized tonic clonic seizures and vocalizations are observed less in children compared to adults. Semiological differences between adults and children may be due to development of frontal lobes along with 
anatomic and functional organization of the maturing brain.

\section{REFERENCES}

1. Fogarasi A, Tuxhorn I, Hegyi M, et al. Predictive clinical factors for the differential diagnosis of childhood extratemporal seizures. Epilepsia 2005; 46: 1280-1285.

2. Hirfanoglu T, Serdaroglu A, Kurt G, et al. Outcomes of resective surgery in children and adolescents with focal lesional epilepsy: The experience of a tertiary epilepsy center. Epilepsy Behav 2016; 63: 67-72.

3. Englot DJ, Breshears JD, Sun PP, Chang EF, Auguste KI. Seizure outcomes after resective surgery for extratemporal lobe epilepsy in pediatric patients. J Neurosurg Pediatr 2013; 12: 126-133.

4. Rasmussen T. Characteristics of a pure culture of frontal lobe epilepsy. Epilepsia 1983; 24: 482-493.

5. So NK. Mesial frontal epilepsy. Epilepsia 1998; 39: 49-61.

6. Kotagal P, Arunkumar G, Hammel J, et al. Complex partial seizures of frontal lobe onset statistical analysis of ictal semiology. Seizure 2003; 12: 268-281.

7. Luders H, Acharya J, Baumgartner C, et al. Semiological seizure classification. Epilepsia 1998; 39: 1006-1013.

8. O’Brien TJ, Mosewich RK, Britton JW, et al. History and seizure semiology in distinguishing frontal lobe seizures and temporal lobe seizures. Epilepsy Res 2008; 82: 177-182.

9. Saygi S, Katz A, Marks DA, et al. Frontal lobe partial seizures and psychogenic seizures: comparison of clinical and ictal characteristics. Neurology 1992; 42: 1274-1277.

10. Fogarasi A, Janszky J, Faveret E, et al. A detailed analysis of frontal lobe seizure semiology in children younger than 7 years. Epilepsia 2001; 42: 80-85.

11. Jobst BC, Siegel AM, Thadani VM, et al. Intractable seizures of frontal lobe origin: clinical characteristics, localizing signs, and results of surgery. Epilepsia 2000; 41: 1139-1152.

12. Battaglia D, Lettori D, Contaldo I, et al. Seizure semiology of lesional frontal lobe epilepsies in children. Neuropediatrics 2007; 38: 287-291.

13. Sinclair DB, Wheatley M, Snyder T. Frontal lobe epilepsy in childhood. Pediatr Neurol 2004; 30: 169-176.

14. Herman ST, Walczak TS, Bazil CW. Distribution of partial seizures during the sleep--wake cycle: differences by seizure onset site. Neurology 2001; 56: 1453-1459.

15. Loddenkemper $T$, Vendrame $M$, Zarowski $M$, et al. Circadian patterns of pediatric seizures. Neurology 2011; 76: 145-153.

16. Gurkas E, Serdaroglu A, Hirfanoglu T, Kartal A , Yilmaz U, Bilir E. Sleep-wake distribution and circadian patterns of epileptic seizures in children. Eur J Paediatr Neurol 2016; 20: 549-54.

17. Hamer HM, Wyllie E, Luders HO, et al. Symptomatology of epileptic seizures in the first three years of life. Epilepsia 1999; 40: 837-844.
18. Nordli DR Jr, Kuroda MM, Hirsch LJ. The ontogeny of partial seizures in infants and young children. Epilepsia 2001; 42: 986-990.

19. Manford M, Fish DR, Shorvon SD. An analysis of clinical seizure patterns and their localizing value in frontal and temporal lobe epilepsies. Brain 1996; 119: 17-40.

20. Laskowitz DT, Sperling MR, French JA, et al. The syndrome of frontal lobe epilepsy: characteristics and surgical management. Neurology 1995; 45: 780-787.

21. Bonini F, McGonigal A, Trébuchon A, et al. Frontal lobe seizures: from clinical semiology to localization. Epilepsia 2014; 55: 264-277.

22. Bancaud J, Talairach J. Clinical semiology of frontal lobe seizures. Adv Neurol 1992; 57: 3-58.

23. Kim KJ, Lee R, Chae JH, et al. Hwang YS. Application of semiological seizure classification to epileptic seizures in children. Seizure 2002; 11: 281-284.

24. Prevost J, Lortie A, Nguyen D, et al. Nonlesional frontal lobe epilepsy (FLE) of childhood: clinical presentation, response to treatment and comorbidity. Epilepsia 2006; 47: 2198-2201.

25. Janszky J, Fogarasi A, Jokeit H, et al. Lateralizing value of unilateral motor and somatosensory manifestations in frontal lobe seizures. Epilepsy Res 2001; 43: 125133.

26. Bonelli SB, Lurger S, Zimprich F, et al. Clinical seizure lateralization in frontal lobe epilepsy. Epilepsia 2007; 48: 517-523.

27. So EL. Value and limitations of seizure semiology in localizing seizure onset. J Clin Neurophysiol 2006; 23 : 353-357.

28. Chugani HT, Phelps ME, Mazziotta JC. Positron emission tomography study of human brain functional development. Ann Neurol 1987; 22: 487-497.

29. Holthausen H, Hoppe M. Hypermotor Seizures. In Lüders HO, Noachtar S (Eds) Epileptic Seizures Pathophysiology and Clinical Semiology. New York: Churchill Livingstone; 2000: 439-448.

30. Fogarasi A, Tuxhorn I, Janszky J, et al. Age-dependent seizure semiology in temporal lobe epilepsy. Epilepsia 2007; 48: 1697-1702.

31. Lieb JP, Dasheiff RM, Engel J Jr. Role of the frontal lobes in the propagation of mesial temporal lobe seizures. Epilepsia 1991; 32: 822-837.

32. Aso K, Watanabe K, Negoro T, Nakashima S. Frontal lobe epilepsy of childhood onset. Epilepsia 1997; 38(Suppl 6): 40-41.

33. Fogarasi A, Janszky J, Tuxhorn I. Ictal emotional expressions of children with partial epilepsy. Epilepsia 2007; 48: 120-123.

34. Bartolomei F, Trebuchon A, Gavaret M, et al. Acute alteration of emotional behaviour in epileptic seizures is related to transient desynchrony in emotion-regulation networks. Clin Neurophysiol 2005; 116: 2473-2479.

35. Janszky J, Fogarasi A, Jokeit $\mathrm{H}$, et al. Are ictal vocalisations related to the lateralisation of frontal lobe epilepsy? J Neurol Neurosurg Psychiatry 2000; 69: 244-247. 
36. Gallmetzer P, Leutmezer F, Serles W, et al. Postictal paresis in focal epilepsies--incidence, duration, and causes: a video-EEG monitoring study. Neurology 2004; 62: 2160-2164.

37. Horvath R, Kalmar Z, Feher N, Fogarasi A. Gyimesi C, Janszky J. Brain lateralization and seizure semiology: ictal clinical lateralizing signs. Ideggyogy Sz 2008; 61: 231-237.
38. Fogarasi A, Janszky J, Tuxhorn I. Peri-ictal lateralizing signs in children: blinded multiobserver study of 100 children $<$ or $=12$ years. Neurology 2006; 66: 271274.

39. Leutmezer F, Baumgartner C. Postictal signs of lateralizing and localizing significance. Epileptic Disord 2002; 4: 43-48. 